\begin{tabular}{cc}
\hline & \\
4 & $\begin{array}{c}\text { International Journal of Case Reports } \\
\text { (ISSN:2572-8776) }\end{array}$
\end{tabular}

\title{
A Fatal Case of Non- typhoidal Salmonella Pyogenic Pericardial Effusion in an Immunocompetent Adolescent
}

\section{Teh $\mathbf{C Y}^{1}$, Ahmad Kashfi AR ${ }^{2}$}

${ }^{1}$ Department of Internal Medicine, Universiti Sultan Zainal Abidin, Kuala Terengganu.

${ }^{2}$ Infectious Disease Unit, Department of Internal Medicine, Hospital Sultanah Nur Zahirah, Kuala Terengganu.

\section{BACKGROUND}

Salmonella infection is common in tropical countries including Malaysia. It is invasive in immunocompromised and those of extreme ages. It typically presents with gastrointestinal symptoms such as diarrhea, abdominal pain or vomiting. Extra-intestinal manifestations are seen in $30 \%$ of salmonellosis cases. These atypical manifestation leads to difficulty and delay diagnosis thus poorer outcome. Pericardium involvement is estimated to be less than $2 \%$ of all cases and has mortality rate as high as $50 \%{ }^{1}$. As high as $70 \%$ of pericarditis cases were identified to be immunosuppressed ${ }^{2}$; these include chronic immunosuppressant usage, autoimmune disease, end stage renal failure, malignancy and etc. Herein, we report a fatal case of pyogenic pericardial effusion by Salmonella enteritidis in an immunocompetent adolescent.

\section{CASE PRESENTATION}

A 16-years-old Malay boy was referred from GP to our centre in April 2015 with CXR finding suggestive of pericardial effusion. He had prolonged cough for 8 months, associated with breathlessness and failure symptoms. He had on and off fever but denied gastrointestinal symptom. He had multiple visits to private practitioners and was investigated for Tuberculosis, which yielded negative result. His condition continued to deteriorate.

*Correspondence to Author:

Teh CY

Department of Internal Medicine, Universiti Sultan Zainal Abidin, Kuala Terengganu.

\section{How to cite this article:}

Teh CY, Ahmad Kashfi AR. A Fatal Case of Non- typhoidal Salmonella Pyogenic Pericardial Effusion in an Immunocompetent Adolescent. International Journal of Case Reports, 2019 4:62 
Of note, he had no significant medical and surgical illness. There was no history of contact with tuberculosis patients, recent travelling history or high risk behaviour.

Upon admission, he was tachypnea and in shock. His blood pressure was $90 / 56 \mathrm{mmHg}$ with a pulse rate of $102 \mathrm{bpm}$. His JVP was raised and heart sound was muffled. Lung examination revealed reduced breath sound bilaterally with generalized rhonchi. Initial blood investigations revealed leukocytosis with predominant neutrophils $\left(16 \times 10^{9} / \mathrm{L}\right)$. ABG showed type 1 respiratory failure. ESR was raised, $100 \mathrm{~mm} /$ hour. Chest $X$-ray showed a congested lung field consistent with pulmonary oedema. Bedside echocardiogram revealed a large pericardial effusion with diastolic right atrium and ventricle collapsed, indicating temponade effect. Emergency pericardialcentesis drained 1.3L of frank pus. IV Augmentin (Amoxicillin and calvulanate potassium) was empirically started.

Pericardial fluid culture grew Salmonella Enteritidis which was sensitive to ceftriaxone and ciprofloxacin, thus antibiotic was switch to Ceftriaxone. Despite given targeted therapy, his general condition did not improve; pericardial pus re- accumulates, causing temponade thus requiring second drainage. Antibiotic was switch to ciprofloxacin and later meropenem in worried of poor pericardium penetration. Average daily drainage was 25 to $35 \mathrm{ml}$. Repeated Echocardiogram showed loculated collection of pus in pericardium. HIV screening and autoimmune screenings were negative. Full blood picture was normal. No screening of malignancy done.

His condition continued to deteriorate, requiring ventilator and inotropic support. He succumbed to death on third week of admission.

\section{DISCUSSION}

Salmonella is a group of Gram-negative bacilli bacteria, typically divided into classical Typhoid and Non-typhoidal serotype (NTS). NTS usually infects broad spectrum of animals and infrequently, may infect human. As it is transmitted via faecal oral route, large outbreak usually occurred in poor hygiene environment and improper food handling ${ }^{1}$. Salmonella infection in healthy individual leads to activation of pro-inflammatory mediators and thus secretion of cytokines in local intestinal mucosa. Hence diarrhoea is a defence mechanism in healthy individual ${ }^{8,} 9,10$. Salmonella bacteraemia occurred as a result of alteration in local intestinal mucosal immunity, mainly due to reduced lymphocytes and $\lg A$ secreting plasma cells ${ }^{7}$.

Not so often, it may manifest with extraintestinal symptom when it got restricted to respective organs. Salmonell pericarditis typically occurred in immune-suppressed patients. Cardiovascular salmonellosis is extremely rare, which can present as endocarditis, myopericarditis, pericarditis or endovascular infection6. Salmonella pericarditis is rare and deadly, with only 30 cases reported so far world wide1. Most reported cases were prior to antibiotic advancement era. Salmonella pericarditis usually presents with cardiac or pulmonic symptomatology: dyspnea (73\%), fever $(47 \%)$, chest pain or discomfort (31\%), cardiac palpitations (21\%) and pericardial temponade $(53 \%)^{2}, 3,4$. Due to its rarity and atypical presentation, diagnosis often delayed.

Our patient was unwell for prolonged period of time (8 months) without gastrointestinal symptoms, thus lead to the suspicion of underlying immune-suppressed state. However, his infection screening, full blood picture and lactate hydrogenase (LDH) yielded negative result. He denied constitutional symptoms and supplement/traditional medication consumption.

Close hemodynamic monitoring and ECG of small QRS complex may suggest presence of pericardial effusion. Echocardiography remained the best tool in confirming the clinical suspicion. Diagnostic and therapeutic pericardialcentesis is vital as organism often detected in either pericardial fluid culture or gram staining of pericardial fluid ${ }^{5}$. Salmonella 
Enteritidis (58\%) is the commonest organism causing pericarditis, followed by Salmonell Typhimurium $(26 \%)^{2}$, which consistent with our case report. This may be due to the raised prevalence of Salmonella serogroup D and their unique virulence traits ${ }^{11}$.

As pyogenic pericardial effusion has high mortality rate, aggressive treatment is crucial. European Society of Cardiology suggested empirical antibiotic combination of antistaphylococcal and aminoglycoside, followed by targeted antibiotic as per culture result. Antibiotic penetration in pericardium is good. Frequent rinsing with urokinase or streptokinase was suggested in order to liquefy fibrin and purulent exudate. They emphasized open surgical drainage is the preferred method, especially in those with dense adhesions, loculated and thick purulent effusion, recurrence of tamponade, persistent infection, and progression to constriction ${ }^{12}$. As for our patient, surgical drainage should be performed in view of persistent infection, loculated purulent effusion and recurrent temponade.

\section{LEARNING POINTS}

1. Pyogenic Non-typhoidal Salmonella Pericarditis is a rare but deadly disease.

2. Aggressive treatment is crucial.

3. Surgical drainage is preferred over pericardiocentesis.

4. Search for underlying primary illness.

\section{ACKNOWLEDGEMENT}

We would like to thank DG of Health for giving us permission for this publication.

\section{REFERENCES}

1. Manisa $S$, Sistla $S$ etc al. Pericardial effusion- an unusual manifestation of salmonellosis: a case report. Cases J.2008;1:375.

2. Daniel O, Eric MS etc al. Nontyphoidal Cardiac Salmonelolosis: Two case reports and review of the literature. 2014;41(4):401406.

3. Hsu RB, Lin FY. Risk factors for bacteraemia and endovascular infection due to nontyphoid salmonella: a reappraisal. QJM. 2005;98(11):821-827.

4. 10. Cohen PS, O'Brien TF, Schoenbaum SC, Medeiros AA. The risk of endothelial infection in adults with salmonella bacteremia. Ann Intern Med. 1978;89(6):931-932.

5. Doig J.C., Hilton C.J., Reid D.S. Salmonella: a rare cause of subacute effusiveconstrictive pericarditis. $\mathrm{Br}$. Heart $\mathrm{J}$. 1991;65(5):296-297.

6. Gaurav C, Ruby J, Kumar $S$ etc al. Pericardial salmonella with cardiac tamponade and ventricular wall rupture: a case report. 2016;7:83-86

7. Fernandez Guerrero ML, Aguado JM, Arribas A, Lumbreras C, de Gorgolas M. The spectrum of cardiovascular infections due to Salmonella enterica: a review of clinical features and factors determining outcome. Medicine (Baltimore) 2004;83(2):123-38.

8. Hsu RB, Tsay YG, Chen RJ, Chu SH. Risk factors for primary bacteremia and endovascular infection in patients without acquired immunodeficiency syndrome who have nontyphoid salmonellosis. Clin Infect Dis. 2003;36(7):829-34.

9. Muller AJ, Hoffmann C, Galle M, Van Den Broeke A, Heikenwalder $M$, Falter $L$ et al. The S. Typhimurium effector SopE induces caspase- 1 activation in stromal cells to initiate gut inflammation. Cell Host Microbe. 2009;6(2):125-36.

10. Brown M, Eykyn SJ. Non-typhoidal Salmonella bacteraemia without gastroenteritis: a marker of underlying immunosuppression. Review of cases at St. Thomas' Hospital 1970-1999. J Infect. 2000;41(3):256-9.

11. $\mathrm{CC}$ Kuo, $\mathrm{WL} \mathrm{Yu}, \mathrm{CH}$ Lee et al. Purulent constrictive pericarditis caused by Salmonella enteritis in a patient with adultonset still's disease. Medicine (Baltimore). 2017; 96(50): e8949.

12. Maisch B, Seferovic PM, Risitc AD, et al. Guidelines on the diagnosis and management of pericardial diseases executive summary. The Task force on the diagnosis and management of pericardial diseases of the European society of cardiology. Eur Heart J 2004;25:587-610.



IJCR: http://escipub.com/international-journal-of-case-reports/ 